\title{
Homology modeling of thermostable YdaP enzyme from Bacillus licheniformis
}

\author{
Joseph Daniel Wani Lako ${ }^{1}$, Kenneth L. L. Sube ${ }^{2}$, Jada P. Yengkopiong ${ }^{3}$, Clara S. G. Lumori ${ }^{1}$, \\ Justin B. Tongun ${ }^{2}$, Don A. Cowan ${ }^{4}$
}

\begin{abstract}
${ }^{1}$ Department of Biotechnology, School of Applied and Industrial Sciences, University of Juba, Juba, South Sudan, ${ }^{2}$ Department of Biochemistry, School of Medicine, University of Juba, Juba, South Sudan, ${ }^{3}$ Department of Biotechnology, College of Science and Technology, Dr. John Garang Memorial University of Science and Technology, Jonglei State, Bor Town, South Sudan, ${ }^{4}$ Center for Microbial Ecology and Genomics, University of Pretoria, Pretoria, South Africa
\end{abstract}

\begin{abstract}
Bacillus licheniformis YdaP gene encodes for pyruvate oxidase (EC: 1.2.3.3), a key enzyme which catalyzes the oxidative decarboxylation of pyruvate into acetate and $\mathrm{CO}_{2}$. The objective of this study is to predict the YdaP protein structure, by comparison with known X-ray structures and using bioinformatics tools. The three-dimensional model structure of the B. licheniformis YdaP enzyme was constructed using the sequence of $L$. plantarum POX as the template. The model structure of B. licheniformis YdaP showed positional conservation of amino acid residues Asp313 and Ala314, compared with other members of the pyruvate oxidase family. The model structure of B. licheniformis YdaP showed that residues Met466, Ile467 and Glu470 were located on an a-helix connecting to loops in the active cavity. These residues are presumably critical for the catalytic activity of pyruvate oxidases, and have been proposed to be involved in substrate binding. The overall topology of the B. licheniformis YdaP was similar to known pyruvate oxidase crystal structures. The structure of the ThDP motif was identical to that found in the other pyruvate oxidases. However, analysis of the substrate binding cavity showed one major difference. Bulky hydrophobic amino acid residues Tyr469, His476 and Tyr479 formed part of active site cavity. In L. plantarum POX, these correspond to amino acid residues Trp479, Ile480 and Glu483. This observation suggested that these residues would negatively influence the accessibility of large substrates (e.g., aromatic) into the catalytic center. This information may assist in studies aimed at engineering the catalytic active site of the enzyme to improve accessibility of larger substrates to the active site.
\end{abstract}

Keywords: Active site, Bacillus licheniformis, Homology modeling, Protein structure, Pyruvate oxidase, Three-dimensional structure, YdaP enzyme

\section{INTRODUCTION}

The Bacillus licheniformis YdaP gene encodes for pyruvate oxidase (POX) (EC: 1.2.3.3) which is a peripheral membrane associated flavoprotein dehydrogenase that belongs to the thiamine diphosphate-dependent enzymes (Cronan, 1995). The thiamine diphosphate-dependent enzymes catalyze the oxidative decarboxylation of pyruvate to acetate and $\mathrm{CO}_{2}$ (Lako et al., 2018). These enzymes are present in a variety of microorganisms from diverse ecosystems including Escherichia coli (Mather et al., 1982), Corynebacterium glutamicum (Schreiner and Eikmanns, 2005), Staphylococcus aureus (Patton et al., 2001; Zhang et al., 2017), Aerococcus viridans (Juan et al., 2007), and Lactobacillus plantarum (Sedewitz et al., 1984; Goffin et al., 2006; Lorquet et al., 2004). These enzymes have been widely studied due to their importance in biotechnological applications (Tomar et al., 2003) and have been well characterized (Lako et al., 2018). The interest in the POX (Pox; 1.2.3.3) is fueled by its potential capacity to produce important commodity chemicals including acetate in the presence of oxygen and inorganic phosphate. This enzyme requires thiamine diphosphate (ThDP), Flavin adenine diphosphate (FAD), and $\mathrm{Mg}^{2+}$ cofactors for its function in catalyzing the oxidative decarboxylation of pyruvate generating acetate (Tittmann et al., 2005). Characterization of the YdaP enzyme has revealed that it is typically composed of four identical subunits in their native state, with each subunit containing one molecule of the $\mathrm{Mg}^{2+}$ cofactor and ThDP. The subunit of this enzyme forms a lose dimer with ThDP and
Doi: $10.30954 / 2319-5169.01 .2020 .1$
Submission: 23-05-2020
Acceptence: 27-06-2020
Received: $24-05-2020$
Published: $30-06-2020$

*Corresponding author: Joseph Daniel Wani Lako, University of Juba, School of Applied and Industrial Sciences, Department of Biotechnology, Central Equatoria State, Juba Town, South Sudan; Phone: +211 924372730/+211 910083372; E-mail: jlako24@gmail.com 


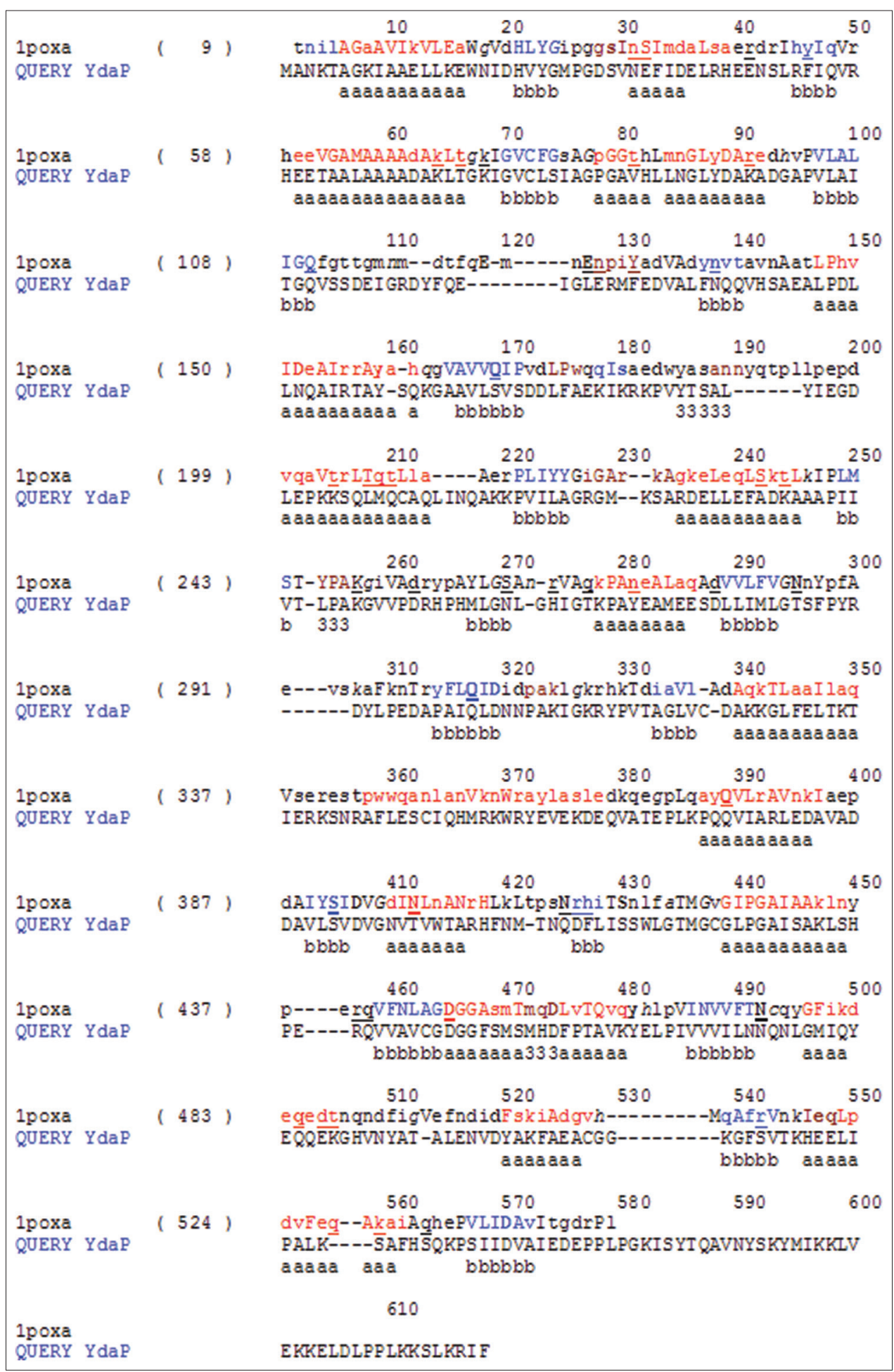

Figure 1: A secondary structure alignment of Bacillus licheniformis $\mathrm{YdaP}$ and Lactobacillus plantarum pyruvate oxidase (1 poxa). The alignment was used to generate the B. licheniformis YdaP 3D model

tight homotetramer in the presence of FAD. The enzyme has been expressed and purified and shown to have a molecular weight of $252 \mathrm{KDa}$ (Lako et al., 2018), with seeming stability in purified form in the presence of ThDP. Several structured studies have been performed with the goal of defining the molecular basis of the functions of the family of these proteins
(Johnson and Overigton, 1993; Muller et al., 1994; Juan et al., 2007). Most revealing were the two reports describing crystal structures of the POX (L. plantarum, PDB: accession number 2EZ9; Aerococcus viridian, and PDB: accession number IV5E). Comparative analysis of these structures reveals that the overall fold is conserved (Juan et al., 2007). The closest 
structural homologue was identified as POX, PDB: 2EZ9 from L. plantarum, which shared $35 \%$ sequence identity to $\mathrm{YdaP}$ (Z-score $>6.0$ ) [Figure 1]. As a result PDB: 2EZ9 was selected as a template for the modeling of $B$. licheniformis $\mathrm{YdaP}$ protein. This enzyme represents one of the first moderately thermophilic Bacillus 9A that expresses a highly active, thermostable protein. YdaP enzyme exhibited a very wide range of substrate specificity. The gene encoding this enzyme has been cloned, expressed, purified, and characterized extensively (Lako et al., 2018). Furthermore, the structure shows extensive interactions in the subunit-subunit interface which is significantly different from the other group of POX and might be responsible for the variation in biochemical properties between the species (Muller et al., 1994). In this study, the three-dimensional structures of the POX: 2EZ9 and YdaP were generated using the homology modeling techniques (Sutcliffe et al., 1987; Martin et al., 1994; Muller et al., 1994; Sănchez and Săli, 1997) to compare the quaternary structures of these bacterial POXs with respect to the enzyme substrate interaction and subunit-subunits interface that might be related to the different biochemical characteristics (Bowie and Eisenberg, 1991).

\section{MATERIALS AND METHODS}

\section{Modeling of the YdaP Enzyme B. licheniformis and POX of $L$. plantarum}

The sequence alignment of the deduced amino acids of B. licheniformis YdaP and the L. plantarum POX (GenBank: B. licheniformis, accession number: CBE70488 and

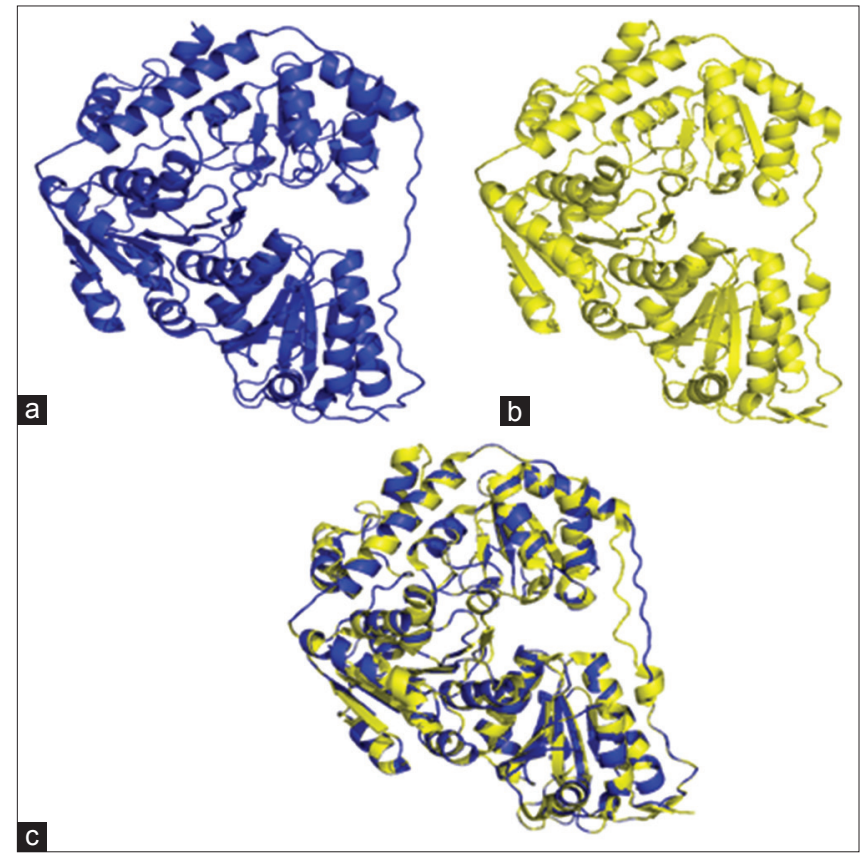

Figure 2: (a) Cartoon representation of the 3D crystal structure of the Lactobacillus plantarum pyruvate oxidase subunit; (b) homology structure model of Bacillus licheniformis YdaP subunit; (c) superimposed structural models of $L$. plantarum POX and B. licheniformis YdaP 3D
L. plantarum, accession number: P 37063, respectively) [Figure 1] were used for model building using the MODELLER 9v4 program (Blundell et al., 1987; Šali and Blundell, 1993; Šali and Overigton, 1994; ; Šali et al., 1995) The closest structural homologue was identified as PDB: 2EZ9 from L. plantarum, which shared 35\% sequence identity to YdaP (Z-score > 6.0) (Figure 2A). As a result PDB: 2EZ9 was selected as a template for the modelling of B. licheniformis $\mathrm{YdaP}$ protein [Figure 2B]. A superimposition of the structural model of $B$. licheniformis $\mathrm{YdaP}$ with $L$. plantarum POX shows overall structural similarity [Figure 2c]. YdaP shares $35 \%$ sequence identity with $L$. plantarum. The assessment and validation of the model stereochemistry were carried out using the RAMPAGE (Lovell et al., 2001) software. This program analyses and plots $\Psi$ and $\varnothing$ angles in the structure. The $\Psi, \varnothing$ plot for the model structure is considered as a reliable method of evaluating torsion angles and has become an important strategy for validation of protein model structures (Kleywegt and Jones, 1998). Overall $\Psi, \varnothing$ distribution in B. licheniformis $\mathrm{YdaP}$ structure was shown to be good [Figure 3].

\section{RESULTS AND DISCUSSION}

\section{Description of the Model Structure}

The $B$. licheniformis YdaP model suggested that the protein monomer was comprised three distinct domains, separated by loops [Figure 4]. These domains were identified using homology search of the secondary structure alignment between B. licheniformis YdaP and L. plantarum POX. The entire globular structure consisted of $22 \alpha$-helices and 21 $\beta$-sheets. The $\mathrm{N}$-terminal domain was commenced with a

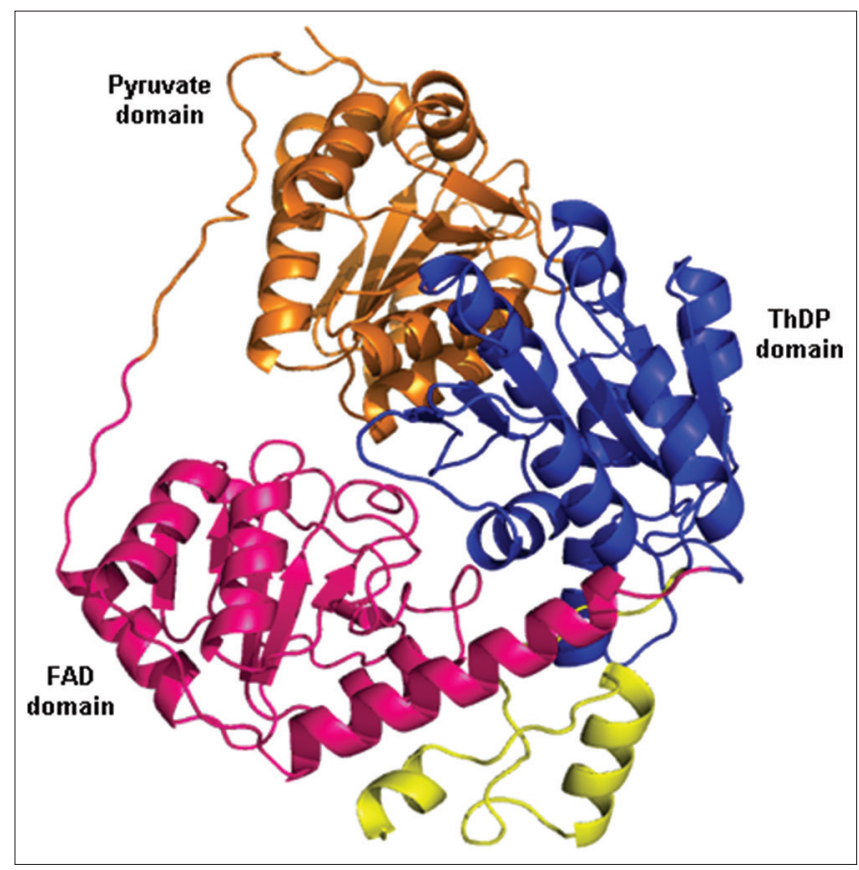

Figure 3: Cartoon representation of the YdaP subunit 
long core domain stretching from residues 3-183 (in orange). This was followed by the FAD domain, which spans residues 184-351 (in pink). These domains lead to the long ThDP domain, stretching between residues 352 and 550 (in blue). The C-terminal primary structure consists between residues 550 and 572 (in yellow) was thought to be a membrane anchor (Neumann et al., 2008). These features are found in all elucidated POX structures (Muller and Schulz, 1993; Neumann et al., 2008; Wille et al., 2006).

The cofactor FAD was predicted to bind to the YdaP enzyme at Asp313 and Ala314 in the FAD domain [Figure 5], which corresponded to residues Asp323 and Ala324 in L. plantarum POX and appeared to be conserved in both structures. This prediction was based on the fact that these residues were highly conserved in POXs from different organisms (Wille et al., 2006).

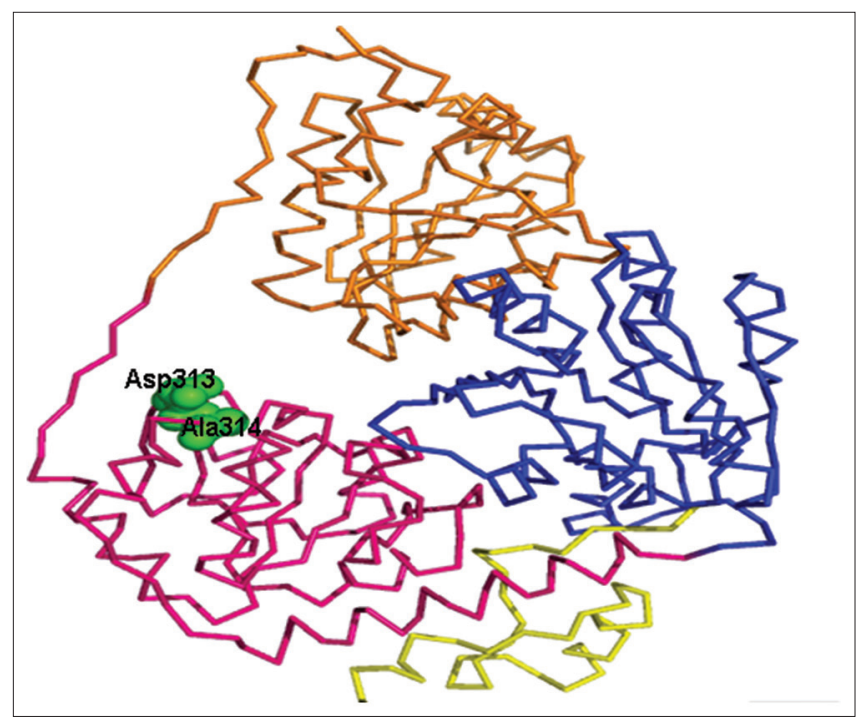

Figure 4: Ribbon representation of residues Asp313 and 314 (in green) predicted to be involved in binding to the flavin adenine diphosphate cofactor

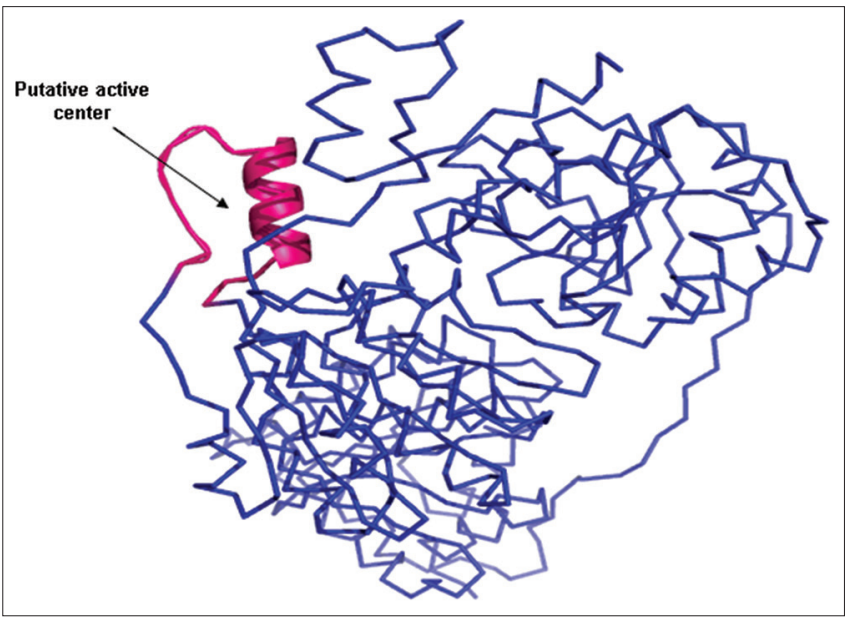

Figure 5: Proposed active center of Bacillus licheniformis YdaP

\section{Topological Description of the YdaP Model}

The model structure of YdaP displayed an overall topology similar to the experimentally determined structures of POX from L. plantarum (Bajorath et al., 1994; Wille et al., 2006), $E$. coli, and $A$. viridans (Juan et al., 2007). The active site structure showed considerable topological homology in both models and template structures (Sánchez and Šali, 1997; Shi et al., 2001). However, the YdaP substrate-binding pocket was similar to the equivalent site in L. plantarum POX, supporting experimental data showing that $\mathrm{YdaP}$ accepted pyruvate and some larger branched chain substrates (Lako et al., 2018). The ThDP motifs of both model structures exhibited a similar structure, their sequences homology of $52 \%$. This observation suggests that the two enzymes are closely related and could indicate an evolutionary relationship (Arnold, 1998) [Figure 6].

\section{Disulfide Bridges}

A detailed analysis of the overall model structure of B. licheniformis YdaP performed using the SSprov 4.5

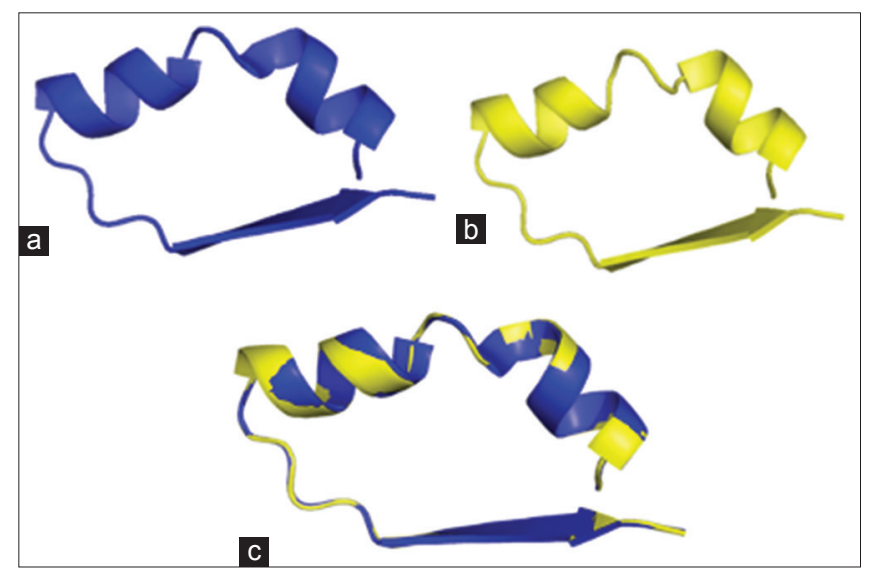

Figure 6: Cartoon representation of (a) ThDP motif of YdaP from Bacillus licheniformis; (b) ThDP motif of $L p P O X$ from Lactobacillus plantarum; (c) superimposed ThDP motifs of both structure models

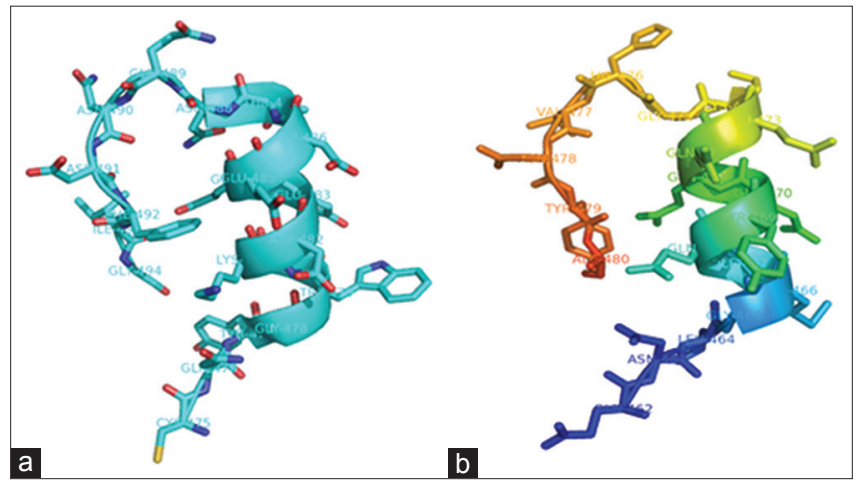

Figure 7: Representation of active center of (a) Lactobacillus plantarum pyruvate oxidase; (b) Bacillus licheniformis YdaP and residues thought to be involved in catalytic activity 
SCRATCH protein predictor (/www.ics.uci.edu/ baldig/ scratch/explanation.html) revealed the presence of seven cysteine residues (C72, C196, C312, C337, C411, C432, and C495). Cysteines C312 and C337 and C411 and C432 were predicted to form disulfide bonds. However, the $\mathrm{YdaP}$ structure model predicted the distances between these cysteine residues to be approximately $3.5 \AA$ Á, compared to $2.0 \AA \hat{A}$ for typical disulfide bonds, suggesting that they do not form the disulfide bonds in vivo. It is known that disulfide bonds serve the critical function of stabilizing protein fold. Furthermore, it also plays a key role in oxidative, heat, and toxic element stresses (Leichert et al., 2003).

\section{Catalytic Site Residues}

The B. licheniformis YdaP showed considerable homology to the L. plantarum POX in the catalytic center [Figure 7]. Putative active site residues were identified in the YdaP model structure [Table 1], the putative active center was made up of a $\alpha$-helix and two loops (Johnson et al., 1994; Jones, 1999), which were located at the subunit interface (Rapp and Friesner, 1999). In the model structure of $B$. licheniformis YdaP, the extended curved accessible channel was identified [Figure 7] and this channel was suggested to allow the accessibility of the substrate into the active site. The amino acid residues of this channel region were relatively less conserved $(20 \%)$ in the structures of both YdaP (target sequence) and POX from L. plantarum (template sequence) (Wille et al., 2006). The residues Ile538, Ser539, Tyr540, Thr541, Val544, and Asn545 were potentially involved in the YdaP substrate binding site [Figure 8]. However, in L. plantarum POX these residues were located in different positions compared to B. licheniformis YdaP. There were considerable differences in the conservation of the substrate binding residues including Lys554, Leu555, Arg556, Leu557, Ala560, and Met561 [Figure 8], which were found in L. plantarum POX (Wille et al., 2006).

The 3D structural model of YdaP of B. licheniformis was constructed based on the closest similarity to the experimentally determined structure of $L p$ POX (Johnson et al., 1994; Wille et al., 2006). The structural model generated was assessed and it revealed to be in a good agreement with template structure suggesting the accuracy spectrum of the $B$. licheniformis YdaP model.

Active site residues were shown to be conserved among the two proteins except for Met 466 of $B$. licheniformis $\mathrm{YdaP}$ which correspond to Trp479 in L. plantarum POX [Table 1] as well as in other homologues; however, the substrate binding residues have low conservation. The residues are clearly localized at the interface of the $B$. licheniformis YdaP subunit. Inspection of the active site residues revealed that Met466, Ile467, and Gln471 were located in the active site cavity. The active site residues were found to be similar in B. licheniformis YdaP compared to the L. plantarum POX [Table 1] which may possibly allow accessibility of other substrates. The classical features of B. licheniformis YdaP, include ThDP motif signature, catalytic active site, and disulfide bonds, were predicted and evaluated.

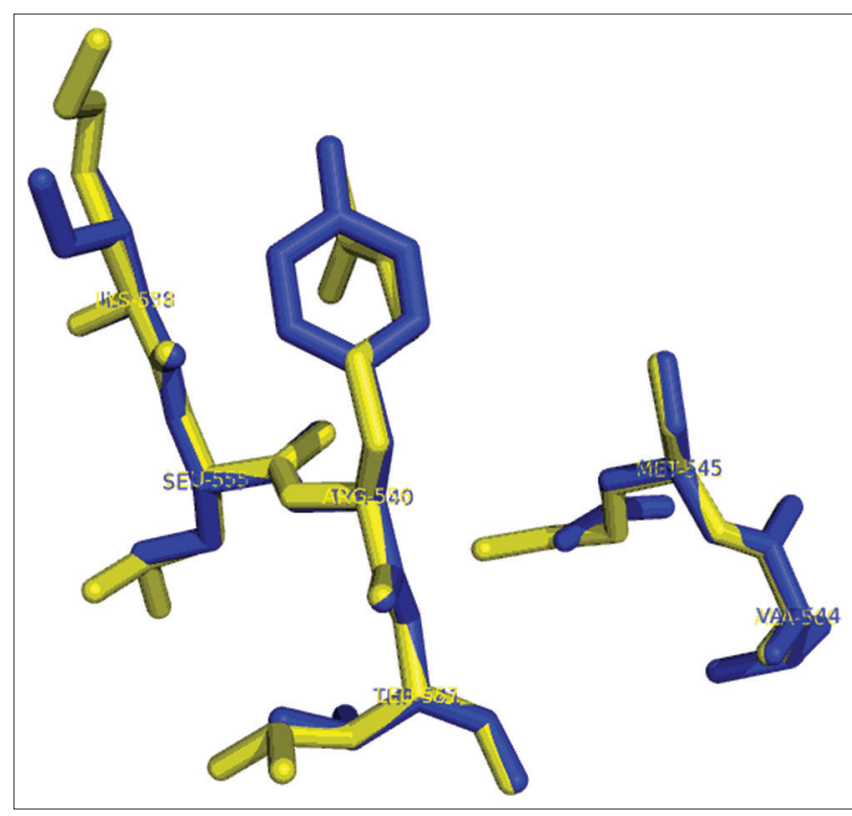

Figure 8: Stick representation of superimposition of residues involved in substrate binding site of Bacillus licheniformis YdaP (in blue) and Lactobacillus plantarum pyruvate oxidase (in yellow)

Table 1: Comparison of active site residues between Lactobacillus plantarum POX and Bacillus licheniformis $\mathrm{YdaP}$

\begin{tabular}{ll}
\hline LpPOX & YdaP \\
Glu59 & Glu52 \\
His89 & His82 \\
Phe121 & Phe114 \\
Gln122 & Gln115 \\
Val376 & Val364 \\
Trp479 & Met466 \\
Ile480 & Ile467 \\
Glu484 & Glu471 \\
\hline
\end{tabular}

POX: Pyruvate oxidase

The YdaP model structure suggests that the catalytic cavity comprised Met466, Ile467, Gln468, Gly469, Lys470, Gln471, Gln472, and Glu473 which were located on the $\alpha$-helix of the active site cavity, while residues Lys474, Gly475, His476, Val477, Asn478, Tyr479, and Ala480 were present on the loop on the opposite site of the catalytic cavity. Overall comparison of the both structures of B. licheniformis YdaP and L. plantarum POX, beside the arrangement of the active center residues showed that there was some level of variation on residues conservation. However, the active center exhibited consistency on structural basis compared to the $L p \mathrm{POX}$ template structure [Figure 7]. Interestingly, the model structure prediction did not provide adequate details of catalytic mechanism of this group of enzymes. Therefore, crystal structure data are required to elucidate on the catalytic mechanisms of YdaP protein. The presence of seven cysteine residues within the YdaP protein 
suggested that the $B$. licheniformis YdaP could form disulfide bonds. However, distance prediction of disulfide bonds by the B. licheniformis YdaP structure model was not appropriate for formation of the disulfide bonds $(\sim 2.0 \AA$ ) $)$. Despite the utilization of the detergent ( $1 \%$ Triton X-100) for the YdaP enzyme activation, there was no role played by it as reducing agent. Therefore, it can be concluded that Triton X-100 did not affect the formation of the disulfide bonds (Lako et al., 2018).

\section{CONCLUSION}

The comparative modeling strategy could provide useful information for improving the characteristics of the YdaP protein, particularly in the identification of the active site residues [Table 1]. The YdaP model structure showed that Met466 Ile467 and Glu471 located in the catalytic cavity are believed to be involved in catalytic activity. However, Glu52, His82, Phe114, and Gln115 appeared to be located on the surface, played an important role in catalytic activity of $B$. licheniformis YdaP. These predictions provided some basic information that could be useful for future studies of particular residues which might be a potential target for site-directed mutagenesis studies for the improvement of the activity of the enzyme (Arnold, 1998).

\section{ACKNOWLEDGMENT}

The authors gratefully would like to acknowledge TMO Renewable and the National Research Foundation (NRF), South Africa for the financial support of this project and without these support we would not have made this study successful.

\section{Financial Support and Sponsorship}

Nil.

\section{Conflicts of Interest}

The authors declare that there is no conflict of interest in this work.

\section{REFERENCES}

Arnold, F.H. 1998. Design by directed evolution. Acc. Cashem. Res., 31, 125-131.

Bajorath, J., Stenkamp, R. and Aruffo, A. 1994. Knowledge-based model building of proteins: Concepts and examples. Protein Sci., 2, 1798-1810.

Blundell, T.L., Sibanda, B.L., Sternberg, M.J.E. and Thornton, J.M. 1987. Knowledge-based prediction of protein structure and the design of novel molecules. Nature, 326, 347-352.

Bowie, J.U., Lüthy, R. and Eisenberg, D. 1991. A method to identify protein sequences that fold into a known three-dimensional structure. Science, 253, 164-170.

Chang, Y.Y. and Cronan, J.E., Jr. 1995. Detection by site-specific disulfide cross-linking of a conformational change in binding of Escherschia coli pyruvate oxidase to lipid bilayers. J. Bio. Chem. 270, 7896-7901.
Goffin, P., Muscariello, L., Lorquet, F., Stukkens, A., Prozzi, D., Sacco, M., Kleerebezem, M. and Hols, P. 2006. Involvement of pyruvate oxidase activity and acetate production in the survival of Lactobacillus plantarum during the stationary phase of aerobic growth. Appl. Environ. Microbiol., 72, 7933-7940.

Johnson, M.S. and Overigton, J.P. 1993. A structural basis for sequence comparisons: An evaluation of scoring methodologies. J. Mol. Biol., 233, 716-738.

Johnson, M.S., Srinivasan, N., Sowdhamini, R. and Blundell, T.L. 1994. Knowledge-based protein modelling. Crit. Rev. Biochem. Mol. Biol., 29, 1-68.

Jones, D.T. 1999. GenTHREADER: An efficient and reliable protein folds recognition method for genomic sequences. J. Mol. Biol., 287, 797-815.

Juan, E.C.M., Hoque, M.M., Hossain, M.T., Yamamoto, T., Imamura, S., Suzuki, K., Sekiguchi, T. and Takénaka, A. 2007. The structures of pyruvate oxidase from Aerococcus viridans with cofactors and with a reaction intermediate reveal the flexibility of the active-site tunnel for catalysis. Acta Crystallogr. Sect. F Struct. Biol. Cryst. Commun., 63, 900-907.

Kleywegt, G.J. and Jones, T.A. 1998. Phi/psi-chology: Ramachandran revisited. Structure, 4, 1395-1400.

Lako, J.D.W., Yengkopiong, J.P., Stafford, W.H.L., Tuffin, M. and Cowan, D.A. 2018. Cloning, expression and characterization of thermostable YdaP from Bacillus licheniformis 9A. Acta Biochim. Pol., 65, 59-66.

Leichert, L.I.O., Scharf, C. and Hecker, M. 2003. Global characterization of disulfide stress in Bacillus subtilis. J. Bacteriol., 185, 1967-1975.

Lorquet, F., Goffin, P., Muscariello, L., Baudry, J.B., Ladero, V., Sacco, M., Kleerebezem, M. and Hols, P. 2004. Characterization and functional analysis of the poxB gene, which encodes pyruvate oxidase in Lactobacillus plantarum. J. Bacteriol., 186, 3749-3759.

Lovell, S.C., Davis, J.W., Arendall, W.B. $3^{\text {rd }}$, de Bakker, P.I., Word, J.M., Richardson, M.G. and Richardson, J.S. 2001. Structure validation by Calpha geometry phi, psi and Cbeta deviation. Proteins, 50, 437-450.

Martin, A.C.R., MacArthur, M.W. and Thorton, J.M. 1997. Assessment of comparative modeling in CASP2. Proteins, 1, 14-28.

Mather, M., Schopfer, L.M., Massey, V. and Gennis, R.B. 1982. Studies of the flavin adenine dinucleotide binding region in Escherichia coli pyruvate oxidase. J. Biol. Chem., 257, 12887-12892.

Muller, Y.A. and Schulz, G.E. 1993. Structure of thiamine and flavindependent enzyme pyruvate oxidase. Science, 259, 965-967.

Muller, Y.A., Schumacher, G., Rudolph, R. and Schulz, G.E. 1994. The refined structures of a stabilized mutant and of wild-type pyruvate oxidase from Lactobacillus plantarum. J. Mol. Biol., 237, 315-335.

Neumann, P., Weidner, A., Pech, A., Stubbs, M.T. and Tittmann, K. 2008. Structural basis for membrane binding and catalytic activation of the peripheral membrane enzyme pyruvate oxidase from Escherichia coli. Proc. Nat. Acad. Sci., 105, 17390-17395.

Patton, T.G., Rice, K.C., Foster, M.K. and Bayles, K.W. 2005. The Staphylococcus aureus cidC gene encodes a pyruvate oxidase that affects acetate metabolism and cell death in stationary phase. Mol. Microbiol, 56, 1664-1674.

Rapp, C.S. and Friesner, R.A. 1999. Prediction of loop geometries using a generalized Born model of solvation effect. Proteins, $35,173-183$.

Šali, A. and Blundell, T.L. 1993. Comparative protein modeling by satisfaction of spatial restraints. J. Mol. Biol., 234, 779-815. 
Šali, A. and Overington, J.P. 1994. Derivation of rules for comparative protein modeling from a database of protein structure alignments. Protein Sci., 3, 1582-1596.

Šali, A., Potterton, L., Yuan, F., Vlijmen, H. and Karplus, M. 1995. Evaluation of comparative protein structure modeling by MODELLER. Proteins, 23, 318-326.

Sánchez, R. and Šali, A. 1997. Advances in comparative proteinstructure modeling. Curr. Opin. Struct. Biol., 7, 206-214.

Sănchez, R., Ya, B.A., Feyfant, E. and Šali, A. 1997. Homology protein structure modeling. Trans. Am. Cryst. Assoc., 32, 81-91.

Schreiner, M.E. and Eikmanns, B.J. 2005. Pyruvate: Quinone oxidoreductase from Corynebacterium glutamicum: Purification and biochemical characterization. J. Bacteriol., 187, 862-871.

Sedewitz, B., Schleifer, K.H. and Gotz, F. 1984. Purification and biochemical characterization of pyruvate oxidase from Lactobacillus plantarum. J. Bacteriol., 160, 273-278.

Shi, J., Blundell, T.L. and Mizuguchi, K. 2001. Sequence structure homology recognition using environment-specific substitution tables and structure-dependent gap penalties. J. Mol. Biol., 310, 243-257.

Sutcliffe, M.J., Hanseef, I., Carney, D. and Blundell, T.L. 1987. Knowledge based modelling of homologue proteins. Part I. Three dimensional frameworks derived from the simultaneous superposition of multiple structures. Protein Eng., 1, 377-384.

Tittmann, K., Wille, G., Golbik, R., Weidner, A., Ghisla, S. and Hübner, G. 2005. Radical phosphate transfer mechanism for the thiamin diphosphate and FAD-dependent pyruvate oxidase from Lactobacillus plantarum. Kinetics coupling of intercofactor electron transfer with phosphate transfer to acetyl-thiamin diphosphate via a transient FAD semiquinone/hydroxyethylThDP radical pair. Biochemistry, 44, 13291-13303.

Tomar, A., Eiteman, M.A. and Atman, E. 2003. The effect of acetate pathway mutations on the production of pyruvate in Escherichia coli. Appl. Microbiol. Biotechnol., 62, 76-82.

Wille, G., Meyer, D., Steinmetz, A., Hinze, E., Golbik, R. and Tittmann, K. 2006. The catalytic cycle of a thiamin diphosphate enzyme examined by cryocrystallography. Nat. Chem. Biol., 2, 324-328.

Zhang, X., Kenneth, W. and Bayles, S.L. 2017. Staphylococcus aureus CidC is a pyruvate: Menaquinone oxidoreductase. Biochemistry, 56, 4819-4829.

How to cite this article: Lako, J.D.W., Sube, K.L.L., Yengkopiong, J.P., Lumori, C.S.G., Tongun, J.B. and Cowan, D.A. 2020. Homology modeling of thermostable YdaP enzyme from Bacillus licheniformis. Int. J. Bioinform. Biol. Sci. 8(1), 6-12. 